\title{
Supporting figures and tables
}

\begin{tabular}{|c|c|c|c|}
\hline ITP18 & 1 & & \\
\hline bZIP52 & 1 & & 1 \\
\hline VIP1 & 1 & & \\
\hline bZIP69 & 1 & & \\
\hline POSF21 & 1 & 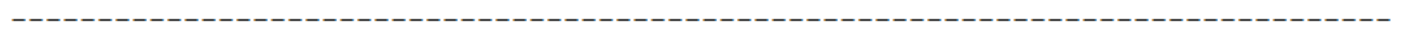 & \\
\hline bZIP29 & 1 & MG-DTEKCNSDMIQRLHSSFGTTSSSIPKNPISQLDLNPNFIRSSAPQFSKPFSDSGKRIGVPPSHPNLIPPTSPFSQIP & 79 \\
\hline bZIP30 & 1 & MGGGGDTTDTNMMQRVNSSSGTSSSSIPKH---NLHLNPALIR-SHHHFRHPFT------GAPPPP---IPPISPYSQIP & 67 \\
\hline UNE4 & 1 & 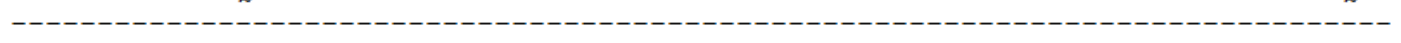 & \\
\hline bZIP31 & 1 & 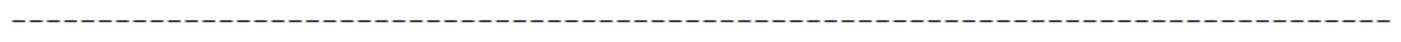 & \\
\hline bZIP33 & 1 & 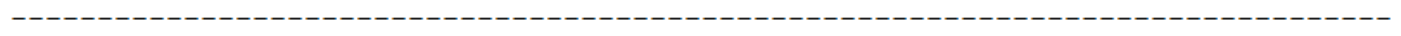 & \\
\hline bZIP74 & 1 & -----MNGRGNMTQYQQNPFSTDDGGQSTGVSLSSRTSLSPPLIRYPAGSPDFSPGPRCTTQPSPTFSDFTQASPSLTSF & 75 \\
\hline bZIP71 & 1 & 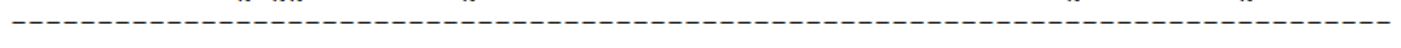 & \\
\hline bZIP18 & 1 & 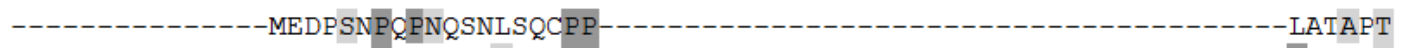 & \\
\hline bZIP52 & 1 & 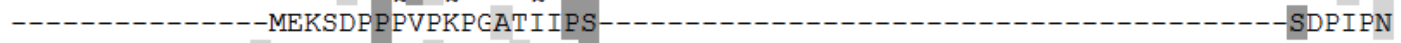 & 25 \\
\hline VIP1 & 1 & 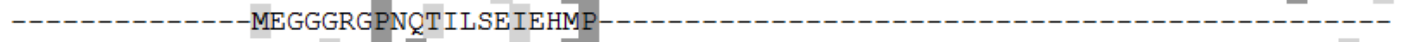 & 20 \\
\hline bZIP69 & 1 & -------------MDKEKSPA PिPPSGGLPPPS--GRYSAFSPNGSS FAMKAESSFPPLTPSGSNSSDANRFSHDISRM & 63 \\
\hline POSF21 & 1 & 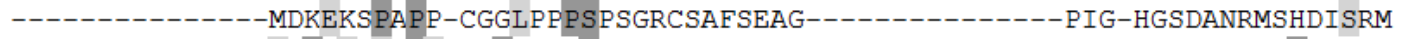 & 48 \\
\hline bZIP29 & 80 & TTRQPGSHNFNPGGANHSRSMSQPNSFFSFDSLPPLSPSPFRDHDVSMEDRDSGVFNSNHSLPPSPFTRCNSTSSSSLRV & 159 \\
\hline bZIP30 & 68 & ATLQP---------RHSRSMSQPSSFFSFDSLPPLNPS-APSVSVSVEEKTGAGFSP--SLPPSPFTMCHSSSSRNAGD & 134 \\
\hline UNE4 & 1 & 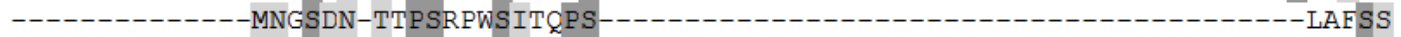 & 24 \\
\hline bZIP31 & 1 & 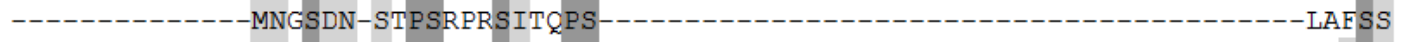 & 24 \\
\hline bzIP33 & 1 & 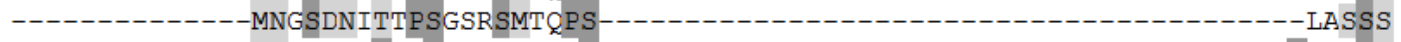 & 25 \\
\hline bZIP74 & 76 & NNPASFTPSFSFSNIHQMIPTPSSSHNSKASVSSASSSSFYFPQTSPSSCSTPSSFSPDSFSHSNTGPWSIPQPSPVFSS & 155 \\
\hline bzIP71 & 1 & -------------MTISYPAKEFLQTIFKTPYLKN-------------------------LYLSPTMSI & 31 \\
\hline bZIP18 & 26 & --------------EVQFRLPEDLDLS---------EPFGGFDELG------SEDDLFC & 69 \\
\hline bZIP52 & 26 & ADPIPSSSFHRRSRSDD------------------MSMEMMFMDPLSSA----------APPS-SDDLP-- ${ }^{---}$SDDDLFS & 71 \\
\hline VIP1 & 21 & EAPRQRISHHRRARSETFFSGESID-----------DLLLFDPSDIDFSSLDFLNAPP PPQQSQQQPQAS PMSVDSEETS & 89 \\
\hline bZIP69 & 64 & 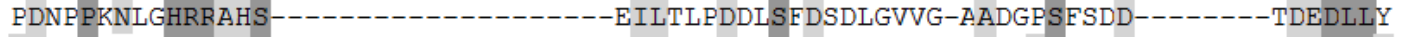 & 114 \\
\hline POSF21 & 49 & LDNPPKKIGHRRAHS-----------------EILTLPDDLSFDSDLGVVGNAADGASFSDE-------TEEDLIS & 100 \\
\hline bZIP29 & 160 & GESLPPRKSHRRSNSDIPSGFNSMP-----LIPPRPLERSFSGGECADWSKSNPFVKKESSCEREGVGER--EAMDDLFS & 232 \\
\hline bzIP30 & 135 & GENLPPRKSHRRSNSDVTFGFSSMMSQNQKSPPLSSLERSISGEDTSDWSNLVKKEPREGFYKGRKPEVE--AAMDDVFT & 212 \\
\hline UNE4 & 25 & 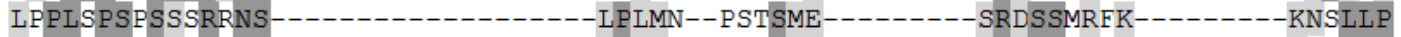 & 65 \\
\hline bZIP31 & 25 & 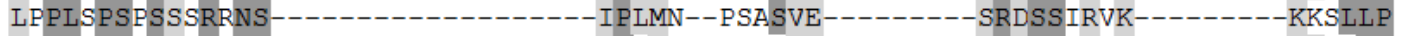 & 65 \\
\hline bzIP33 & 26 & LPPLSPSSSTERRNS-------------------LPSMSFSPSAPVE----------SRDSSIKVN---------KNPSLP & 68 \\
\hline bZIP74 & 156 & IAPASSALSSFGPDSFSHSNTGTWSIP-----QPSPVFSSIAPASSAPPLFGRDSFPRSNRGKGSLIHRS---PVLSVLP & 227 \\
\hline bzIP71 & 32 & YDTLIGSFATTLKRYLLKS--------------DVAVMYTSPSTYVE---------NEDSSFRFN--------QNCSPMP & 80 \\
\hline bZIP18 & 70 & DSAGPSAPRSDNP---------ESAENGGAEAGNSR & 112 \\
\hline bZIP52 & 72 & SEIDVD----------------------------SLTSNPNPFQNPS------LSSSNS---------VSGAANPPPPPSSR & 109 \\
\hline VIP1 & 90 & SNGVVP--------------------------PNSLPPKPEARFGRHVRSF ${ }^{*}$ VDSDFFDDLGVTEEKFIATSSGEKKK & 141 \\
\hline bZIP69 & 115 & 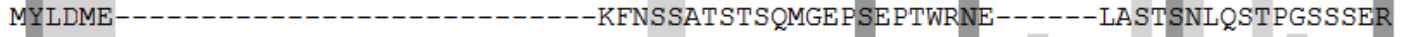 & 160 \\
\hline POSF21 & 101 & 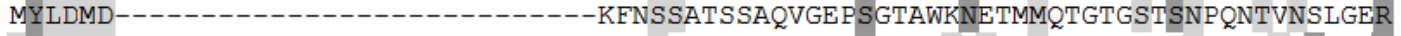 & 152 \\
\hline bZIP29 & 233 & AYMNLENIDVLNSSEADDSKNGNENRDDMES-SRASGTKTNGSDTEGESSSVNESANNNMNSSGEKRESVKRRAAGGDIA & 311 \\
\hline bZIP30 & 213 & AYMNLDNIDVLNSFGGEDGKNGNENVEEMES-SRGSGTKKTNGGSSSDSE-GDSSASGNVKVALSSSSSSGVRRAGGDIA & 290 \\
\hline UNE4 & 66 & 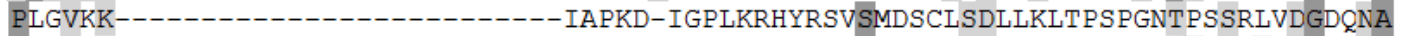 & 118 \\
\hline bZIP31 & 66 & 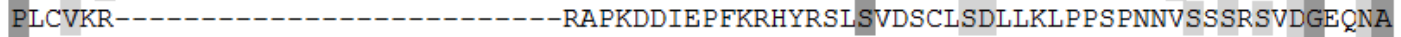 & 119 \\
\hline bZIP33 & 69 & 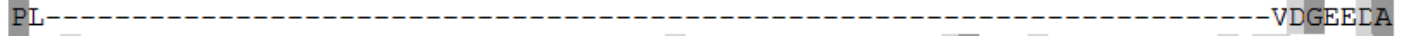 & 77 \\
\hline bZIP74 & 228 & PAPVYSS---------------------------NPMVRSSPPGHPSPSAHLEEMSNRPPLHPQPRVPVTRSNSAKVSGSR & 281 \\
\hline bzIP71 & 81 & PWVLG-------------------------TKSKTI INTLPPKKSHKRTNTDIIGIHSMTSQNSSGSSYFQLLD & 129 \\
\hline bZIP18 & 113 & $\mathrm{P}--\mathrm{R}$ & 144 \\
\hline bZIP52 & 110 & 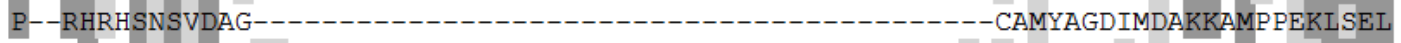 & 144 \\
\hline VIP1 & 142 & GNHHHSRSNSMD GEMSSAS----------------------------FNIESIIASVSGKDSGKKNMGMGGDRIAEL & 190 \\
\hline bZIP69 & 161 & PRIRHQHSQSMDGSTTIKP------------------------------EMLMSGNEDVSGVDSKKAISAAKISEL & 206 \\
\hline POSF21 & 153 & 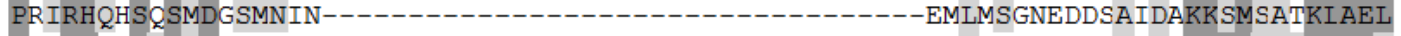 & 197 \\
\hline bZIP29 & 312 & PTTRHYRSVSVDSCFMEKLSFGDES-LKPPPSPGSMSRKVSPTNSVDGNSGAAFSIEFNNGEFTAAEMKKINANDKIAEM & 390 \\
\hline bZIP30 & 291 & PTGRHYRSVSMDSCFMGKLNFGDESSLKLPPSS---SAKVSPTNSGEGNS-SAYSVEFGNSEFTAAEMKKIAADEKIAEI & 366 \\
\hline UNE4 & 119 & SRLEFDANDYTDDELN-------------------- & 145 \\
\hline bZIP31 & 120 & SELEFDTSVYTDDELN---------------------- & 146 \\
\hline bZIP33 & 78 & 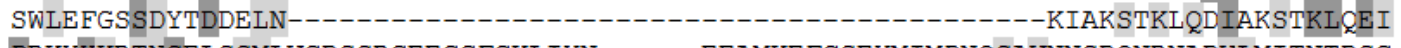 & 113 \\
\hline bZIP74 & 282 & PRKYHKRTNSELSSMLVGDSSRGEEGGFGKLIYN------EEAMKEFCSEYMIMPNQSAVNNSDQNRNADVLMITNTDSG & 355 \\
\hline bZIP71 & 130 & 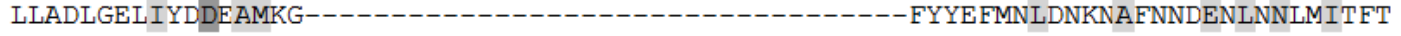 & 174 \\
\hline
\end{tabular}




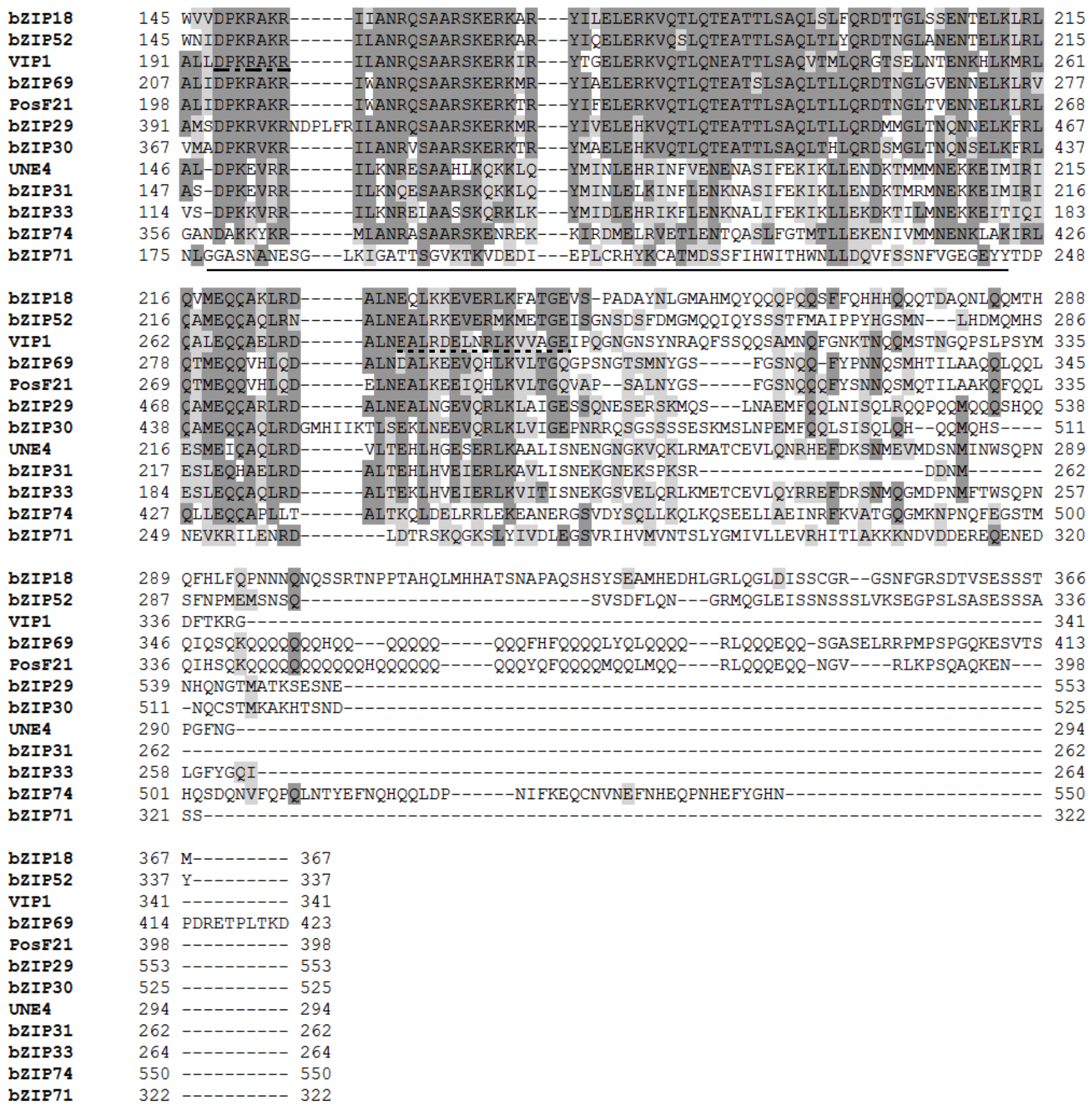

Figure S1. Alignment of the amino acid sequences of Arabidopsis group I bZIP proteins. The amino acid sequences were aligned using Clustal W [47]. Identical amino acid residues conserved in more than four sequences are boxed in dark gray. Similar amino acid residues conserved in more than four sequences are boxed in light gray. The region corresponding to the bZIP domain of VIP1 is shown by the solid underline. The regions corresponding to the NLS and the NES of VIP1 are shown by the dashed underline and the dotted underline, respectively. The bZIP domain, the NLS and the NES of VIP1 were predicted using ELM (Eukaryotic Linear Motif resource: http://elm.eu.org/, [48]). The serine residues at positions 79 and 115 in VIP1 are indicated as *. 


\section{Supporting references}

47. Thompson JD, Higgins DG, Gibson TJ (1994) CLUSTAL W: improving the sensitivity of progressive multiple sequence alignment through sequence weighting, position-specific gap penalties and weight matrix choice. Nucleic Acids Res 22: 4673-4680.

48. Dinkel H, Michael S, Weatheritt RJ, Davey NE, Van Roey K, et al. (2012) ELM--the database of eukaryotic linear motifs. Nucleic Acids Res 40: D242-251. 